\title{
Numerical study of hydrodynamic and thermal behaviors of agitated yield-stress fluid
}

\author{
Moez Hammamia ${ }^{\mathrm{a}}$, Amal Chebbi and Mounir Baccar \\ Computational Fluid Dynamic and Transfer Phenomena, Mechanical Engineering Department, \\ National Engineering School of Sfax, Soukra, B.P. 1173-3038 Sfax, Tunisia
}

Received 24 April 2013, Accepted 11 August 2013

\begin{abstract}
Heat transfer during agitation of Bingham viscoplastic fluid is studied in this paper. The fluid is agitated with an anchor impeller and the heating is made by a jacketed wall of the stirred vessel. Transfers in the agitated vessel, translating hydrodynamic and thermal phenomena, are numerically predicted by means of Computational Fluid Dynamics (CFD) in transient regime. The purpose of this numerical study is to identify the rigid zones and to optimize mixing and heating performances. The Navier-Stokes and energy equations are discretized using finite volume method, and a two-dimensional analysis of the hydrodynamic and transient thermal behaviours generated in the agitated vessel are performed. Fluid rheology is modeled by the Bingham approximation and Papanastasiou's regularization model. Results show the presence of recirculation zones and permit to explain the unpredicted Nusselt number increasing when Oldroyd number increases. This study shows also the importance of the anchor position on the size and the shape of the rigid zones and on the heating performances.
\end{abstract}

Key words: Agitation / Anchor impeller / Viscoplastic fluid / Rigid zones / Heat transfer / Numerical analysis

\section{Introduction}

In many industrial areas mixing is an important step such as the food, pharmaceutical or polymer industries. Mixing operation has to be controlled in order to ameliorate fina1 product properties. Non-Newtonian fluids possibly with a yield stress pose additional problems in agitation. The rheological behavior of these fluids governs the hydrodynamics found in these processes. Other difficulties for the optimization of these processes often occur with the heating of such fluids.

In the aim of controlling the mixing of viscoplastic fluids by a Rushton turbine, many numerical works were made and published: Torrez and Andre [1], Arratia et al. [2], Ein-Mozaffari and Upreti [3]... These works show the existence of well-mixed caverns around the impeller with near stagnant regions outside. By assuming different cavern shapes (spherical, cylindrical, and toroidal), authors have attempted to correlate cavern size to the amount of energy introduced to the system. However the stagnant regions are highly undesirable as they are poorly mixed and they also give rise to poor mass and heat transfer.

The mixing of viscoplastic fluids with anchor impeller has been studied using numerical simulations by Tanguy

\footnotetext{
${ }^{a}$ Corresponding author: HammamiMoez2003@yahoo.fr
}

et al. [4] and Bertrand et al. [5]. In their works the authors present a detailed analysis for highly viscoplastic fluids and observe a weak increase of Metzner-Otto parameter $K_{s}$ (proportionality coefficient between the effective shear rate and the rotation frequency). They also showed the influence of viscoplasticity on the flow pattern and on power consumption.

Later, Anne-Archard et al. [6] have investigated the hydrodynamics and power consumption in laminar stirred vessel flow using numerical computation. The mixing systems investigated are flat-bottomed vessels equipped with double helical ribbon or anchor agitators. In their work, the authors [6] have investigated the distributions of shear rates and their link to power consumption in yield stress fluids. Fully or partially sheared flow situations with plug regions are studied. Anne-Archard et al. [6] have concluded that a constant value for the Metzner-Otto parameter is not a reasonable assumption when the Bingham number varies significantly. In fact, variations in the value of Metzner-Otto coefficient induce differences in the evaluation of the generalized Reynolds number and thus in the determination of the flow regime.

Savreux et al. [7] investigated numerically the detailed flow morphology and the 2D mixing of a viscoplastic fluid in a mixer consisting of a rotating tank and a static anchor impeller. The authors [7] examined the flow structures, 


\section{Nomenclature}

\begin{tabular}{|c|c|c|}
\hline$C_{p}$ & Specific heat & $\mathrm{J} . \mathrm{kg}^{-1} \cdot \mathrm{K}^{-1}$ \\
\hline$d$ & Impeller diameter & $\mathrm{m}$ \\
\hline$\overline{\bar{D}}$ & Rate of strain tensor & $\mathrm{s}^{-1}$ \\
\hline$h$ & Heat transfer cœefficient at the jacketed wall & $\mathrm{W} \cdot \mathrm{m}^{-2} \cdot \mathrm{K}^{-1}$ \\
\hline$k$ & Consistency & Pa.s \\
\hline$l$ & Impeller length & $\mathrm{m}$ \\
\hline$N$ & Rotation speed & $\operatorname{tr} . \mathrm{s}^{-1}$ \\
\hline$P$ & Pressure & $\mathrm{Pa}$ \\
\hline $\boldsymbol{R}$ & Vessel radius & $\mathrm{m}$ \\
\hline rot & Rotor radius & $\mathrm{m}$ \\
\hline$r$ & Radial coordinate & $\mathrm{m}$ \\
\hline$T$ & Temperature & K \\
\hline$T_{0}$ & Ambient temperature & $\mathrm{K}$ \\
\hline$T_{h}$ & Heating temperature & $\mathrm{K}$ \\
\hline$t$ & Time & $\mathrm{s}$ \\
\hline$U, V$ & Velocity components & $\mathrm{m} \cdot \mathrm{s}^{-1}$ \\
\hline \multicolumn{3}{|c|}{ Greek symbols } \\
\hline$\rho$ & Fluid density & $\mathrm{kg} \cdot \mathrm{m}^{-3}$ \\
\hline$\lambda$ & Thermal conductivity & $\mathrm{W} \cdot \mathrm{m}^{-1} \cdot \mathrm{K}^{-1}$ \\
\hline$\tau$ & Second invariant of the stress tensor & $\mathrm{Pa}$ \\
\hline$\tau_{0}$ & Yield stress & $\mathrm{Pa}$ \\
\hline$\overline{\bar{\tau}}$ & Shear stress tensor & $\mathrm{Pa}$ \\
\hline$\dot{\gamma}$ & Shear rate & $\mathrm{s}^{-1}$ \\
\hline \multicolumn{3}{|c|}{ Dimensionless variables } \\
\hline$r=r / R$ & Dimensionless radial coordinate & \\
\hline$R_{1}=\boldsymbol{d} / \mathbf{2} \boldsymbol{R}$ & Dimensionless impeller radius & \\
\hline$m$ & Dimensionless Papanastasiou index & \\
\hline$P=P / \rho(2 \pi N R)^{2}$ & Dimensionless pressure & \\
\hline$t=2 \pi N t$ & Dimensionless time & \\
\hline$T=\left(\boldsymbol{T}-\boldsymbol{T}_{\mathbf{0}}\right) /\left(\boldsymbol{T}_{\mathbf{h}}-\boldsymbol{T}_{\mathbf{0}}\right)$ & Dimensionless temperature & \\
\hline $\bar{T}$ & Averaged dimensionless temperature in the vessel & \\
\hline$U=U / 2 \pi N R$ & Dimensionless radial velocity component & \\
\hline$\vec{V}$ & Dimensionless velocity vector & \\
\hline$V=V / 2 \pi N R$ & Dimensionless tangential velocity component & \\
\hline$Z R$ & Percentage of rigid zones $=$ rigid zone area/area of vessel & \\
\hline$\tau$ & Dimensionless second invariant of the stress tensor & \\
\hline$\dot{\gamma}$ & Dimensionless shear rate & \\
\hline$\eta_{\mathrm{a}}$ & Dimensionless apparent viscosity & \\
\hline$\theta$ & Dimensionless angular coordinate & \\
\hline \multicolumn{3}{|c|}{ Dimensionless numbers } \\
\hline$N u=2 R h / \lambda$ & Nusselt number & \\
\hline$O d=\tau_{0} R /(2 k \pi N R)$ & Oldroyd number & \\
\hline $\operatorname{Pr}=k C_{p} / \lambda$ & Prandtl number & \\
\hline$P e=\boldsymbol{P} \boldsymbol{r} \boldsymbol{R}_{e}$ & Peclet number & \\
\hline$R_{e}=\rho N d^{2} / k$ & Reynolds number & \\
\hline
\end{tabular}

and particularly the static and moving rigid zones induced by the plasticity. It is shown that the increase in rotation velocity is not generally sufficient to reduce the size of the rigid zones. The calculations are made for different types of impeller configurations: the first consisted of chamfered anchor impeller, while two aligned blades and two perpendicular blades were added respectively for the second and the third shapes. In this work power consumption is calculated and the mixing quality is determined by particle tracking.

As we can see, heat transfer to Bingham fluids in mixing process has not been investigated in the previous numerical studies; however there is some works in Newtonian cases. Baccar and Abid [8] characterized numerically the hydrodynamic and heating performances of the anchor and gate agitators utilized for the agitation of 
Newtonian fluid in an agitated vessel. They notice that heat transfer is specially controlled by the turbulence viscosity and is weakly affected by the clearance between the vessel wall and the blade tip of the anchor. Moreover, it appears that the anchor and gate agitators give a similar thermal performance. Also, the evolution in transient regime of the thermal state of the agitated vessel has been studied by Baccar et al. [9] in the case of radial turbine. In this work, the highest value of the local heat transfer coefficient is found at the impeller discharge region where fluid impinges on the wall at the maximum velocity. On the other hand, the change of the heat transfer coefficient is found to be very little with angular position. Nusselt numbers predicted by Baccar et al. [9] are correlated by a dimensionless equation.

Delaplace et al. [10] investigated the heat transfer for several liquids agitated in a rounded bottom vessel equipped with an atypical helical ribbon impeller. In their work, numerical simulations of heat transfer phenomena to highly viscous Newtonian at unsteady states in a jacketed vessel were made using CFD finite volume software. No temperature gradient was detected in the bulk. Also, the authors noted that the thermal boundary layer is established after $30 \mathrm{~s}$ from the beginning of the heating.

Recently, Tutar and Erdogdu $[11,12]$ carried out numerical simulation for heat transfer and velocity field characteristics of two-phase flow systems in axially rotating horizontal cans. Numerical results demonstrated that a little effect of rotation on heating up time is obtained at low speeds of rotation, but a significant effect was observed at higher speeds for air-water phase. Also, the results verified the heat transfer enhancement with higher Coriolis-centrifugal buoyancy force ratio.

In this paper, we present a two-dimensional numerical modelling of the hydrodynamic and transient thermal behaviours in a stirred vessel agitated with anchor impeller. The heat transfer is applied on the jacketed wall of the stirred vessel. The aim of this work was to investigate both mixing and heating of pseudoplastic fluids possessing yield stress by studying the effect of plasticity and impeller position on the hydrodynamic and thermal structures developed in the stirred vessel. For this purpose, the resolution of dynamics and heat transfer equations, governing the transfer phenomena developed in the tank is conducted using finite-volume technique discretization.

\section{Mathematical formulation and numerical method}

In this study, the mixing system consists in a cylindrical vessel equipped with an anchor impeller (Fig. 1). Fluids with yield stress (viscoplastic fluids) are commonly described using the Bingham model:

$$
\begin{cases}\overline{\bar{\tau}}=2\left(\frac{\tau_{0}}{\dot{\gamma}}+k\right) \overline{\bar{D}} \text { if } \quad \tau>\tau_{0} \\ \dot{\gamma} \rightarrow 0 & \text { if } \quad \tau \leq \tau_{0}\end{cases}
$$

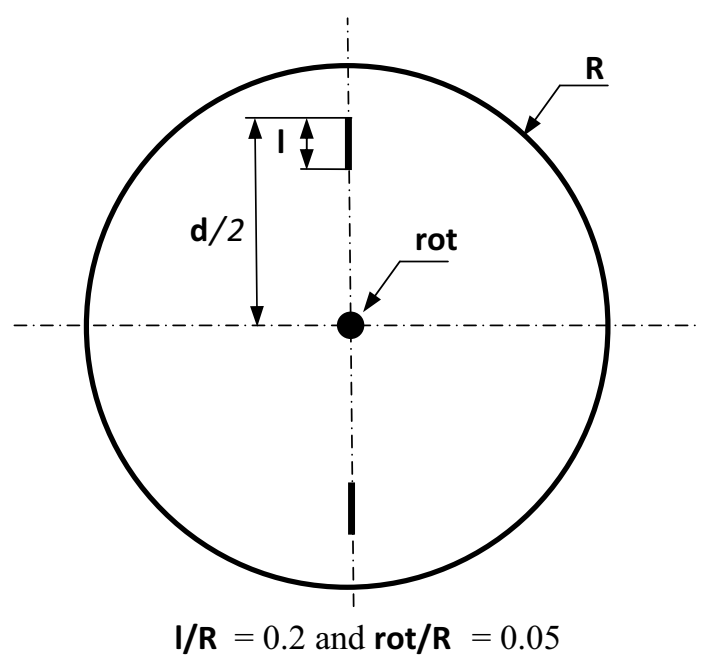

Fig. 1. Geometry of the problem.

In order to simulate the flow of fluid with yield stress Papanastasiou's regularisation model [13] is used (in the dimensionless form):

$$
\tau=\left(1+O d \frac{\left(1-\mathrm{e}^{(-m \dot{\gamma})}\right.}{\dot{\gamma}}\right) \dot{\gamma}
$$

Since the modelling of the mixing system was done in steady state condition, computations were conducted in a reference frame rotating with the impeller. In this case, the motion of this frame requires the introduction of centrifugal and Coriolis forces to the momentum balance equation.

Then, the resulting continuity, momentum and energy coupled equations can be written in $2 \mathrm{D}$ dimensionless form as follow:

Continuity equation:

$$
\operatorname{div} \vec{V}=0
$$

$U$ - velocity component:

$$
\begin{aligned}
\frac{\partial U}{\partial t}+\operatorname{div} & \left(\vec{V} U-\eta_{\mathrm{a}} E \overrightarrow{\operatorname{grad}} U\right)=-\frac{\partial P}{\partial r}-\frac{2}{r} \eta_{\mathrm{a}} \\
& \times E\left(\frac{\partial V}{r \partial \theta}+\frac{U}{r}\right)+\operatorname{div}\left(\vec{S}_{U}\right)+\frac{V^{2}}{r}+r+2 V
\end{aligned}
$$

$V$ - velocity component:

$$
\begin{aligned}
\frac{\partial V}{\partial t}+\operatorname{div}\left(\vec{V} V-\eta_{\mathrm{a}} E \overrightarrow{\operatorname{grad}} V\right) & = \\
-\frac{\partial P}{r \partial \theta}+\eta_{\mathrm{a}} E\left(-\frac{V}{r^{2}}+\right. & \left.\frac{1}{r} \frac{\partial V}{\partial r}+2 \frac{\partial}{r \partial \theta}\left(\frac{U}{r}\right)\right) \\
& +\operatorname{div}\left(\vec{S}_{V}\right)-\frac{U V}{r}-2 U .
\end{aligned}
$$

Energy equation:

$$
\frac{\partial T}{\partial t}+\operatorname{div}\left(\vec{V} T-\left[\frac{\pi}{2}\left(\frac{1}{R_{1}}\right)^{2} P e\right]^{-1} \overrightarrow{\operatorname{grad}} T\right)=0
$$


where

$$
\begin{aligned}
& \vec{S}_{U}=\left(\begin{array}{c}
\eta_{\mathrm{a}} \frac{\partial U}{\partial r} \\
\eta_{\mathrm{a}} r \frac{\partial(V / r)}{\partial r} \\
\eta_{\mathrm{a}} \frac{\partial W}{\partial r}
\end{array}\right), \quad \vec{S}_{V}=\left(\begin{array}{c}
\eta_{\mathrm{a}} \frac{\partial U}{r \partial \theta} \\
\eta_{\mathrm{a}} \frac{\partial V}{r \partial \theta} \\
\eta_{\mathrm{a}} \frac{\partial W}{r \partial \theta}
\end{array}\right) \\
& \eta_{\mathrm{a}}=1+O d \frac{1-\mathrm{e}^{-m \dot{\gamma}}}{\dot{\gamma}} \text { and } E=\left[\frac{\pi}{2}\left(\frac{1}{R_{1}}\right)^{2} R e\right]^{-1} .
\end{aligned}
$$

The above equations are subject to the following initial and boundary conditions.

Initially, the fluid is considered at rest and has ambient uniform temperature. So the dimensionless temperature, pressure and velocities have zero initial values.

Concerning the boundary conditions, the impeller is modelled as infinitely thin surfaces and non-slip conditions are chosen on the tank and on the agitator blades. In the vessel wall, we imposed a fixed dimensionless temperature $T=1$.

The vessel was always assumed to be symmetric along its axis and then it is sufficient to consider only half of the stirred vessel.

The resolution of the equations of continuity, movement and energy, is based on the finite-volume method [14]. The derivatives in the inertia terms were discretized according to the hybrid model and the pressure field was dealt with via a prediction and correction procedure, all described by Patankar [14]. Concerning temporal discretization, an implicit formulation was adopted. The temporal integration has begun using the implicit scheme of alternated directions of Douglass and Gunn [15].

\section{Results and discussion}

In this section, we will present numerical results to give a fine knowledge of the hydrodynamic characteristics and the temporal evolution of the thermal behavior induced in an agitated mixing vessel. A particular interest is provided to study the controlling parameters characterizing the fluid flow and the rigid zones sizes and forms. These parameters are Reynolds number, Oldroyd number and the impeller position.

Next, the results are turned into a fixed frame. The thermal results are given for fixed values of Prandtl number $\left(\operatorname{Pr}=7 \times 10^{3}\right)$, dimensionless impeller length $(\boldsymbol{l} / \boldsymbol{R}=$ $0.2)$ and dimensionless rotor radius $(\operatorname{rot} / R=0.05)$.

\subsection{Rigid zone characteristics}

When the applied stress is beneath a threshold value called the yield stress, rigid zone is obtained and moves like a solid. We note that three types of rigid zones can be detected (Fig. 2):

- Zones Z1: cling to the impeller on both sides.

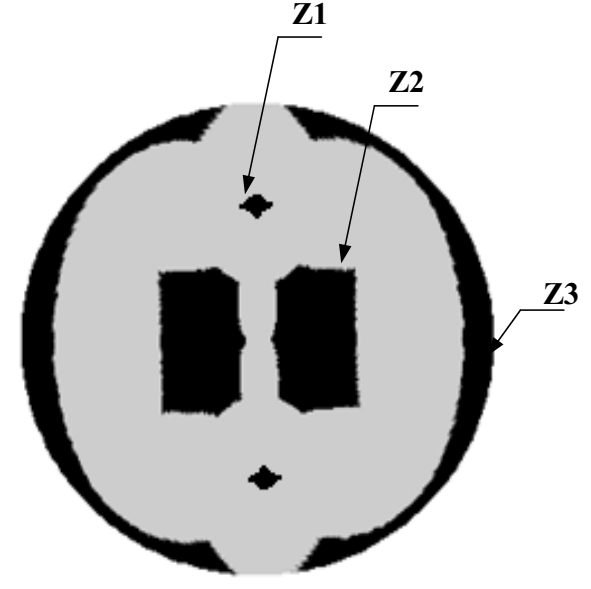

Fig. 2. Typical rigid zones.

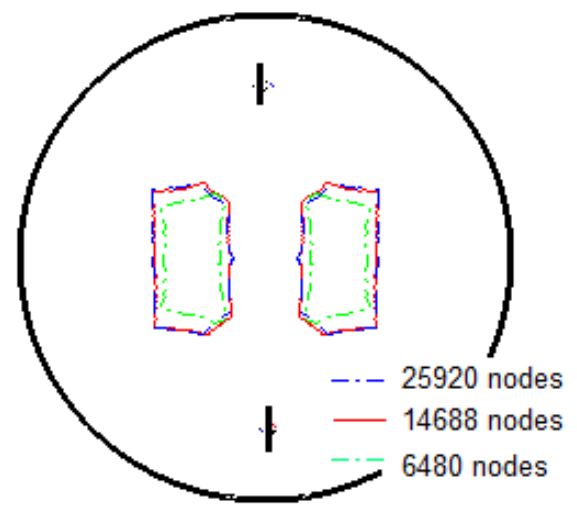

Fig. 3. Mesh sensibility $\left(R_{1}=0.8 ; R e=1\right.$ and $\left.O d=5\right)$.

- Zones Z2: appear on either side of the tank center.

- Zones Z3: appear in the peripheral region cling to the wall of the stirred vessel.

To obtain a precise determination of the rigid zones, the influence of the mesh on the results and in particular on these zones was studied. The grid-independent test is used to determine the minimum grid resolution that achieves a solution independent of the mesh quantities. In this test three different mesh sizes were evaluated: 6480 (coarse mesh), 14688 (fine mesh), and 25920 (very fine mesh). Figure 3 shows that, a computational domain consisting of $108 \times 136$ grid points with uniform grid spacing respectively in radial and angular directions was found to be sufficient for producing accurate results at reasonable computed time.

However, to have more accuracy we must use the very fine mesh (25920) or the mesh must be highly refined near the impeller [7] to obtain the rigid zones Z1 stuck to the impeller. Nevertheless, quantitatively, the overall area of unyielded zones Z1 can be neglected (less than $3 \%$ of the total rigid zone area [7]). On the other hand, it can also be seen in Figure 4 that the effect of these zones on the hydrodynamic behavior is not significant. 


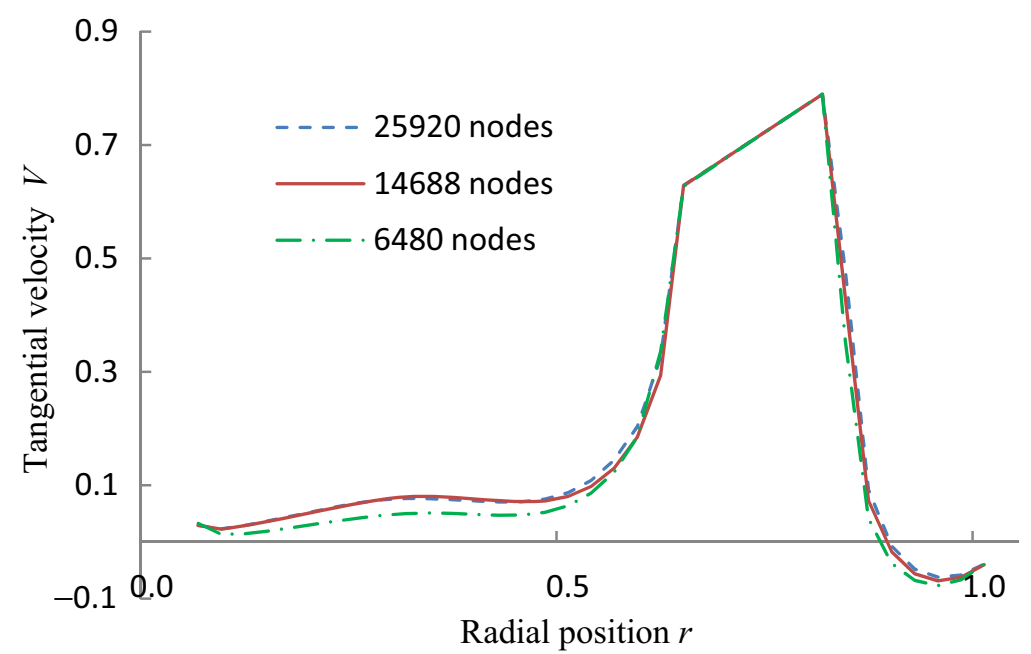

(a)

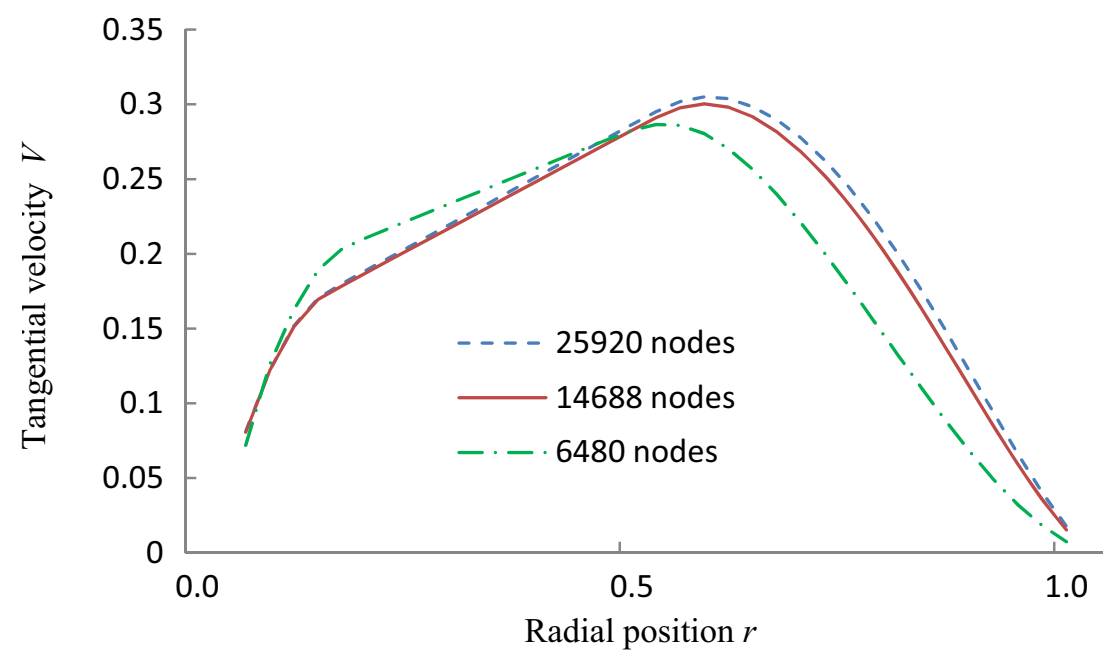

(b)

Fig. 4. Comparison of tangential velocity for different meshes. (a) Comparison of tangential velocity in the impeller plane. (b) Comparison of tangential velocity in the median plane.

\subsection{Yield stress effects}

\subsubsection{Yield stress effect on the hydrodynamic behaviour}

For $R_{1}=0.8$ and $R e=1$, Figure 5 shows the effect of the Oldroyd number on the flow pattern inside the tank. A similar hydrodynamic behaviour to the Newtonian case is observed for $O d=5$, a vortice is formed between the impeller and the wall of the stirred vessel. In these cases the flow is strongly dominated by the tangential component. When $O d=50$, the shape of the hydrodynamic structure changes and a second recirculation zone takes birth nearby the other side of the agitator. Also far from the impeller, when the yield stress increases, the fluid velocity drastically decreases.

To further investigate the effects of viscoplasticity, Figure 6 presents the non-dimensional radial profiles of tangential velocities taken at the median and the impeller planes for $R_{1}=0.8$ and $R e=1$. As soon as plasticity effects are present, a linearity of the tangential velocities profiles is observed in the median plane. This indicates that the fluid exhibits no deformation and solid structures $\mathrm{Z} 2$ are formed. Also for $O d=50$, it is noteworthy that two zones Z3 are formed in the peripheral regions where there is a poor transfer. On the other hand, it can be seen at the impeller plane (Fig. 6a) that recirculation flow becomes.

\subsubsection{Yield stress and inertia effects on the rigid zones}

Figure 7 shows the rigid zones for $R_{1}=0.8$ and different $R e$ and $O d$ numbers. It can be noted that two solid cores Z2 appear on either side of the tank center even for small yield stress. As can be seen in Figure 7, the solid cores Z2 size increases considerably with $O d$. However, the two parts of the peripheral rigid zone Z3 take birth only for high values of $\mathrm{Od}$. This can be explained 


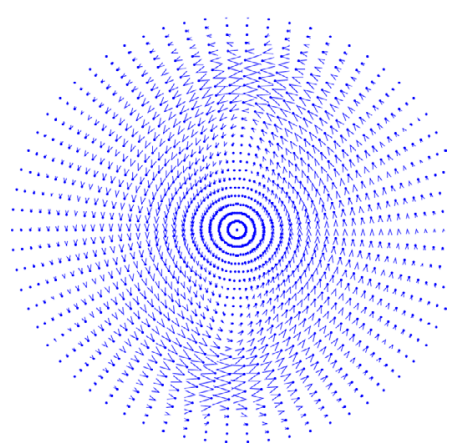

(a)

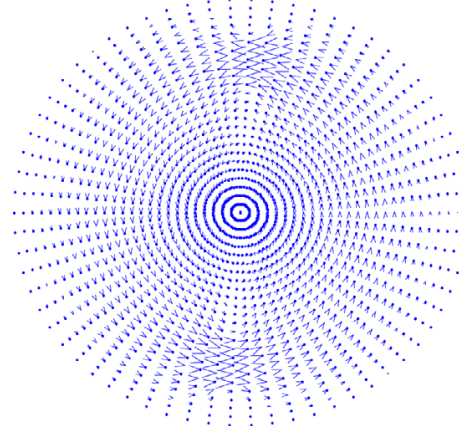

(b)

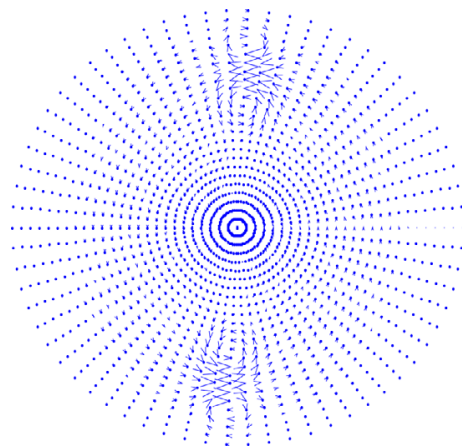

(c)

Fig. 5. Flow patterns induced for $R_{1}=0.8$ and $R e=1$. (a) $O d=0$, (b) $O d=5$, (c) $O d=50$.

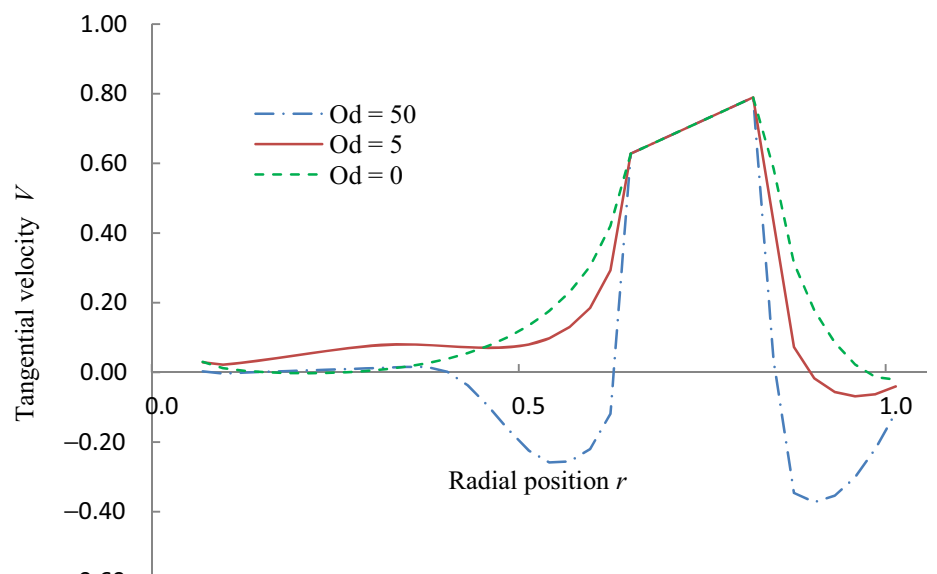

(a)

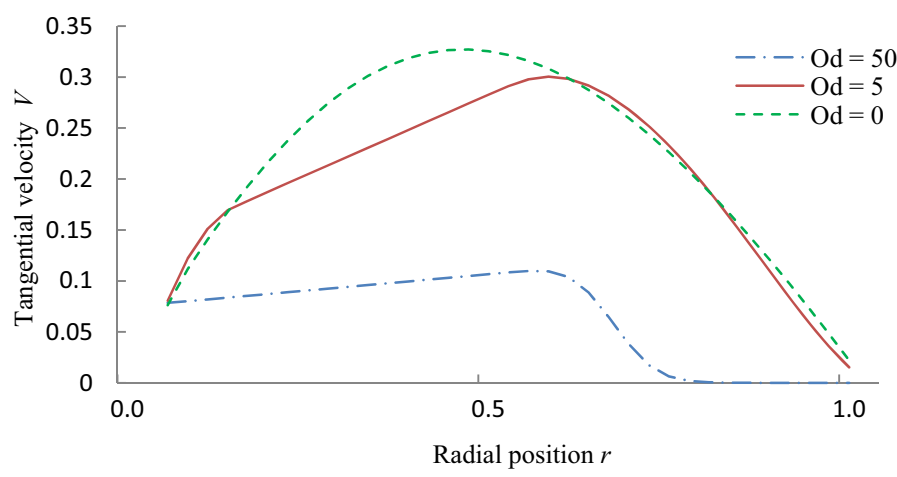

(b)

Fig. 6. Effect of Od number on the tangential velocity $\left(R_{1}=0.8\right.$ and $\left.R e=1\right)$. (a) Comparison of tangential velocity in the impeller plane. (b) Comparison of tangential velocity in the median plane. 


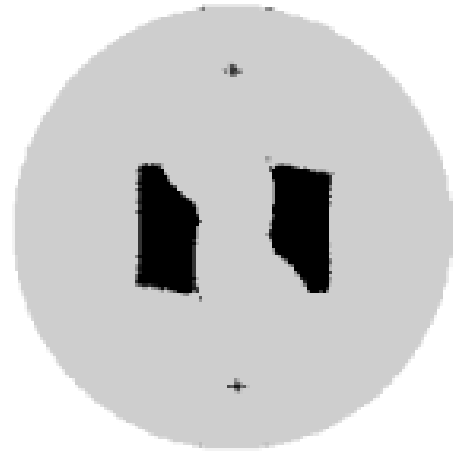

(a)

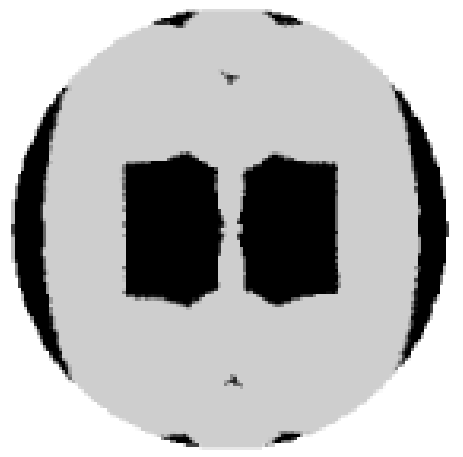

(d)

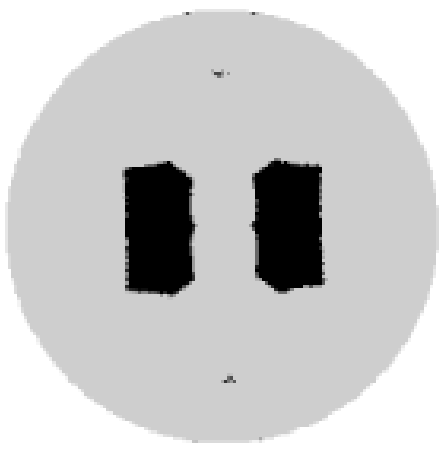

(b)

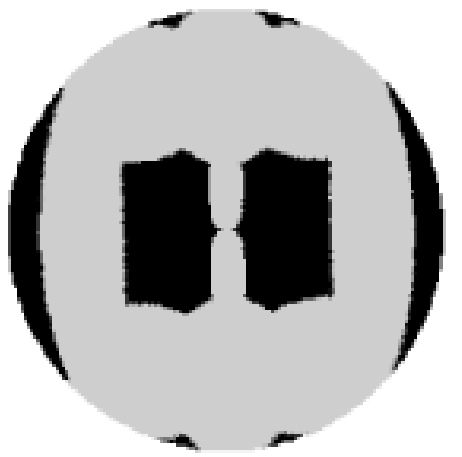

(e)

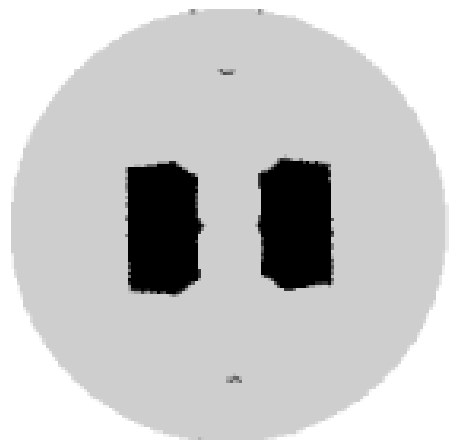

(c)

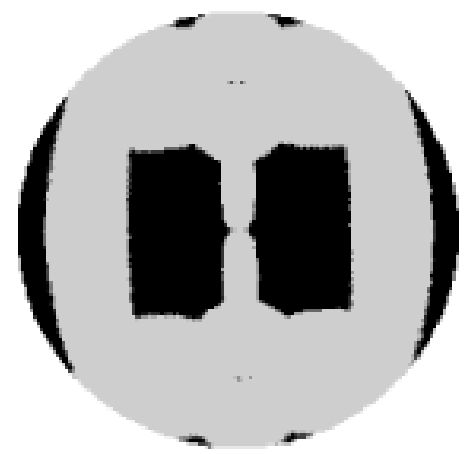

(f)

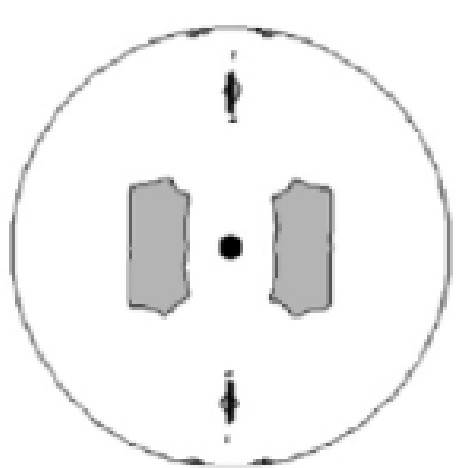

(g)

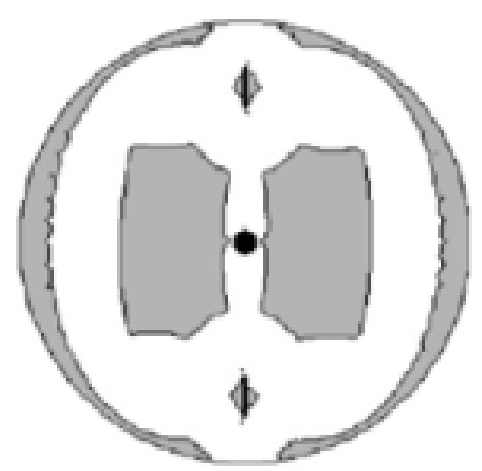

(h)

Fig. 7. Effect of Re and Od on the location and the shape of the rigid zones $\left(R_{1}=0.8\right)$. (a) $R e=20$ and $O d=5 ; Z R=8.69 \%$. (b) $R e=1$ and $O d=5 ; Z R=11.69 \%$. (c) $R e=0.05$ and $O d=5 ; Z R=12.24 \%$. (d) $R e=20$ and $O d=50 ; Z R=27.33 \%$. (e) $R e=1$ and $O d=50 ; Z R=27.48 \%$. (f) $R e=0.05$ and $O d=50 ; Z R=29.12 \%$. (g) $R e=0.05$ and $O d=5$ (Savreux et al. [7]). (h) $R e=0.05$ and $O d=50$ (Savreux et al. [7]).

by the fundamental variation in the structure of the flow for $O d=50$ : the fluid skirts around the impeller and the flow is clearly braked in the peripheral zones (Fig. 5).

If Od number has a strongly effect on the conformation of the rigid zones, however, as it can be seen in Figure 7 , Re number has a negligible influence on the unyielded zones. Also for $O d=5$, the increase in the inertia is not sufficient to reduce the size of the rigid zones. We note that in some specific configurations, especially for a low yield stress value, when $R e=20$ the rigid zones become asymmetrical.

To verify the accuracy of our simulation, a comparison is conducted in Figure 7, where we have reproduced the rigid zones obtained by Savreux et al. [7] for $R e=0.05$ and $R_{1}=0.8$. We can notice a good agreement between our results and those of the previous study.

\subsubsection{Yield stress effect on the thermal behaviour}

To understand better the thermal treatment of mixed Bingham viscoplastic fluid, we have reproduced the thermal behaviour evolution with time for fixed Prandtl number $\left(\operatorname{Pr}=7 \times 10^{3}\right)$. The fluid is heated by a jacketed wall of the stirred vessel. For $O d=50, R e=1$ and $R_{1}$ equal to 0.8 , Figures 8 and 9 show the evolution with time of the temperature distribution in the agitated mixing vessel. The results show the unexpected trend of rapid temperature increasing in the peripheral region. In fact, in the 


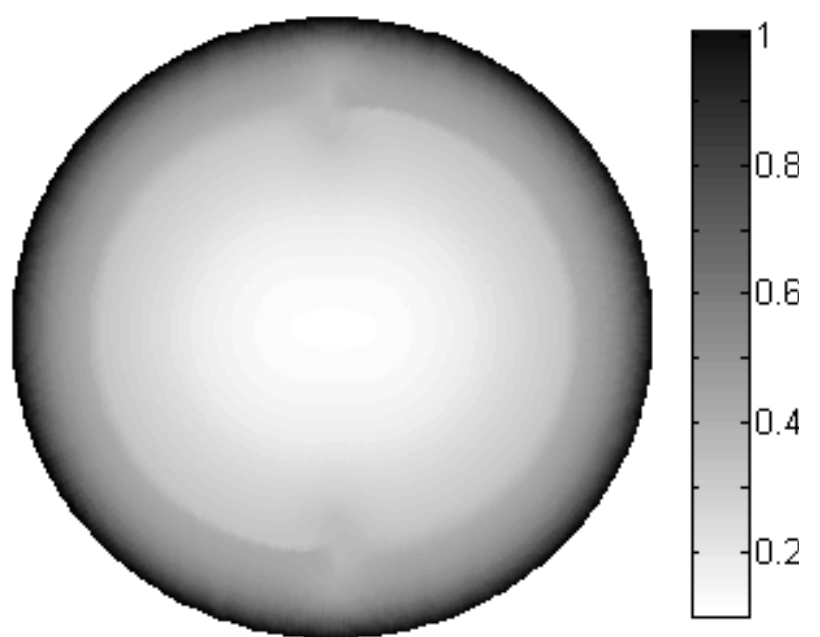

Fig. 8. Temperature distribution at $t=250(O d=50$ and $R e=1)$.

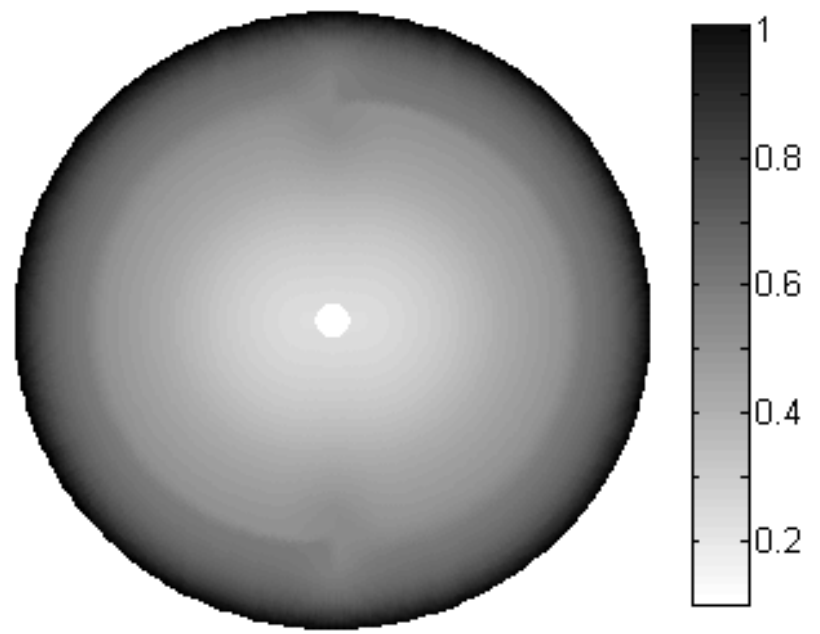

Fig. 9. Temperature distribution at $t=500(O d=50$ and $R e=1)$.

Z3 zones we have a nearly one-dimensional conduction regime, but the vortices, formed between the impeller and the wall of the stirred vessel, enhance the heat transfer. The same phenomenon is observed in the middle of tank where the second recirculation zone improves the transfer.

In order to give more accurate information about the $O d$ number effect on the temperature distribution, Figure 10 represents temperature radial profiles in the median plane at $t=1500$ for $R e=1$. It is found that there is negligible difference between the Newtonian case $(O d=0)$ and $O d=5$. Also for $O d=50$, the effect of the rigid zones $\mathrm{Z} 2$ is not clear. This is can be explained by the fact that the angular rotation speed of the confined fluid is different of the impeller one. So these zones are unlikely to have any impacts for mixing efficiency: there is not a stagnant zone, but the fluid is only sometimes confined in the rigid zones and then the particles can migrate outside these zones and be mixed.

In Figure 11, showing the temporal evolution of the averaged temperature in the mixed tank for $R_{1}=0.8$, we can see that the temperature increases more rapidly when $O d=50$. This unpredicted tendency can be explained by the generation of important vortexes on both sides of the impeller. In fact, conjugated cells improve the fluid homogenisation, and subsequently, convective transfer will be performed.

In order to characterize the instantaneous mean convective heat transfer coefficient through the wall of the agitated vessel, obtained by integrating of the local coefficient over its correspondent exchange surface, we calculate the instantaneous average Nusselt number, defined as follows:

$$
\overline{N u}(t)=\left.\frac{2}{\pi(1-\bar{T})} \int_{0}^{\pi} \frac{\partial T(\theta, t)}{\partial r}\right|_{r=1} \mathrm{~d} \theta .
$$

To study the Oldroyd number effect on the transient heat transfer, the averaged Nusselt number evolution with time is plotted in Figure 12 for various $R e$ and $O d$ numbers and a radius $R_{1}=0.8$. In general, it is found that $N u(t)$ decreases at first and then varies slowly to attend a constant value. The results show the trend of increasing $N u$ with $R e$ number. On the other hand, when $O d=50$ heat transfer is promoted. This tendency is in adequacy with the velocity profile (Fig. 6) that shows that the recirculation flow is intense when the yield stress increases.

\subsection{Effect of the impeller position on the rigid zones}

Figure 13 resumes the effect of the impeller position $R_{1}$ on the location and shape of the rigid zones. Three configurations are studied in this section: $R_{1}=0.7,0.8$ and 0.9 . It can be noticed that the decrease of the radius $R_{1}$ (equal to 0.7 ) can reduce the percentage of rigid zone for the case $O d=5$. However, for $O d=50$ the Z3 zones tend to join together to form a continuous annulus and this configuration prevents the fluid homogenisation. For high $O d$ number, it appears that the configuration with $R_{1}=0.9$ is effective for reducing the rigid zones, in particular Z3.

To verify this behavior, we have reproduced in Figure 14, the average temperature evolution for different radii $R_{1}$ (0.7, 0.8 and 0.9$)$ and $O d$ numbers (5 and 50) with $R e=1$. This figure clearly demonstrates the importance of the impeller position effect on the heat transfer performance. Moreover, it was noted that for $O d=5$ the reduction in rigid zone size when $R_{1}=0.7$ is not a criterion to indicate the heating quality improving. Also, although the same zone rigid percentage is almost obtained for $R_{1}=0.7$ and $R_{1}=0.8$ when $O d=50$, there is a large difference in thermal behaviours. However, it seems that the configuration $R_{1}=0.9$ was the best for agitating and heating viscoplastic fluids. Also, for this configuration there is not a considerable difference between $O d=5$ and 50 cases.

\section{Conclusion}

In this study, the mixing and heating of Bingham viscoplastic fluid in a vessel agitated by an anchor impeller 


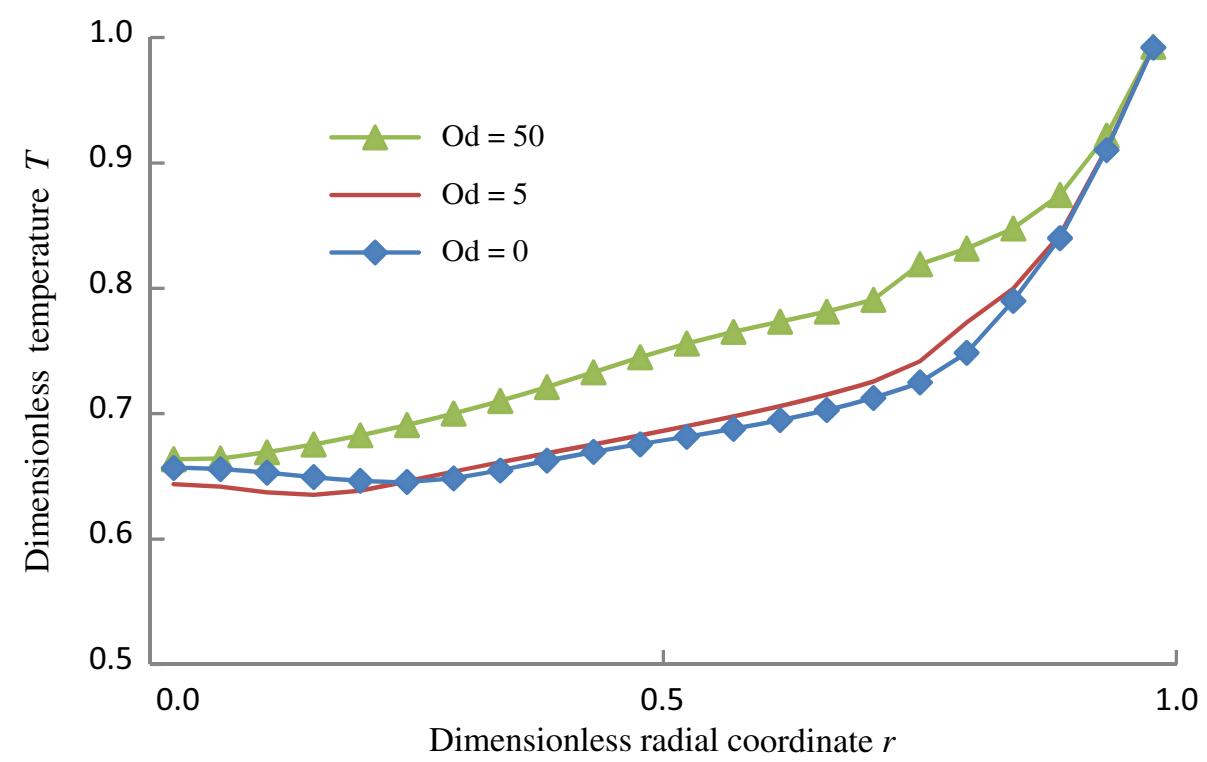

Fig. 10. Effect of Od number on the radial temperature profiles in the median plane at $t=1500$.

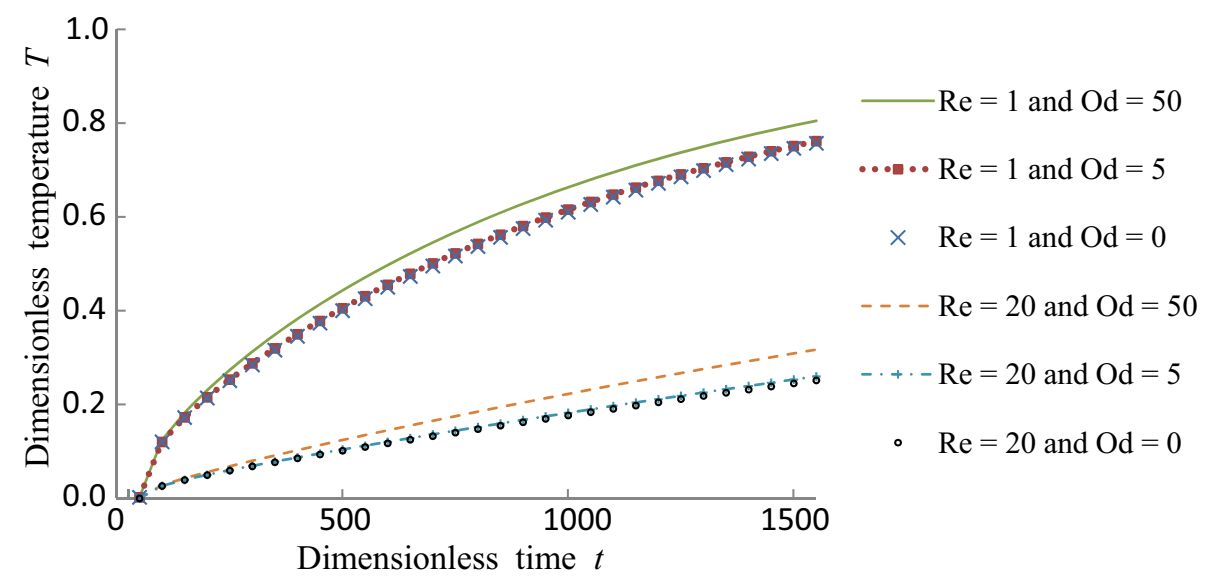

Fig. 11. Effect of Od number on the temporal evolution of the averaged temperature.

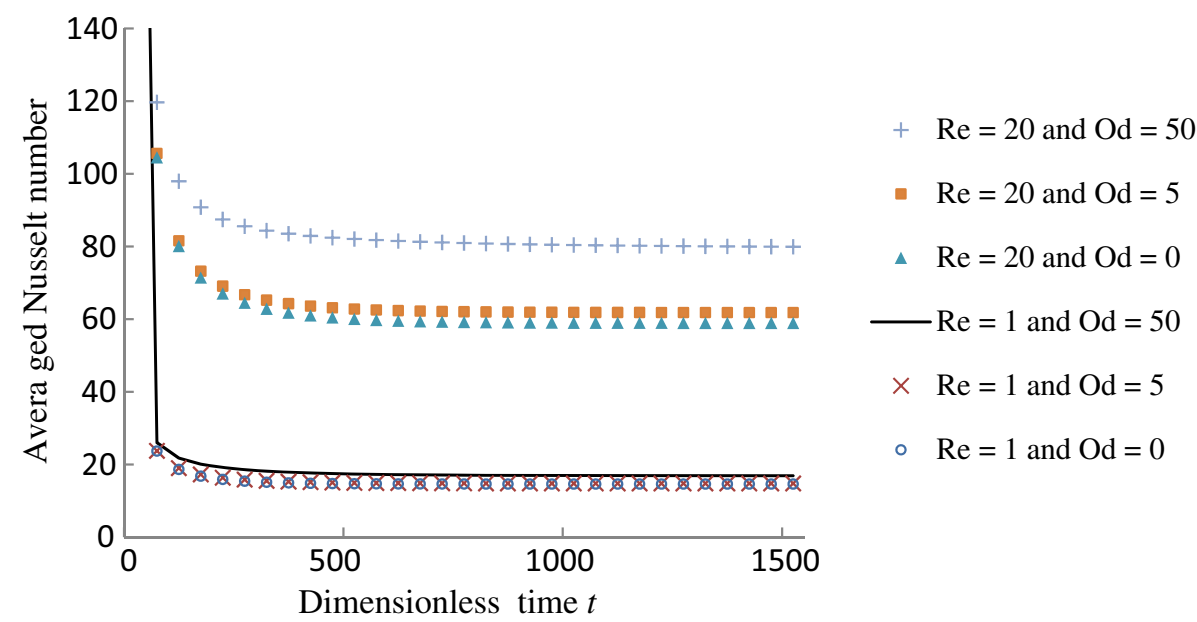

Fig. 12. Temporal evolution of averaged Nusselt number for various Oldroyd numbers. 


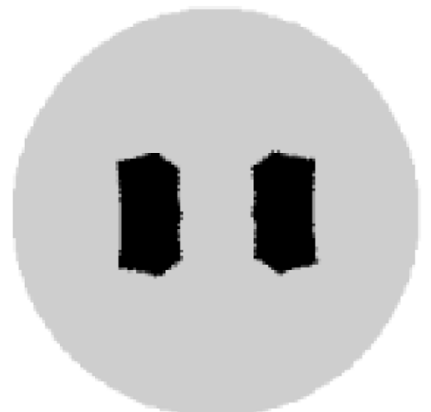

(a)

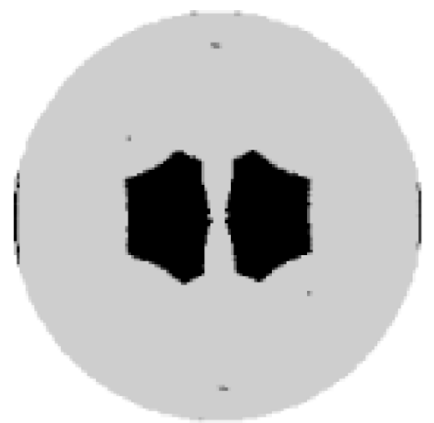

(d)

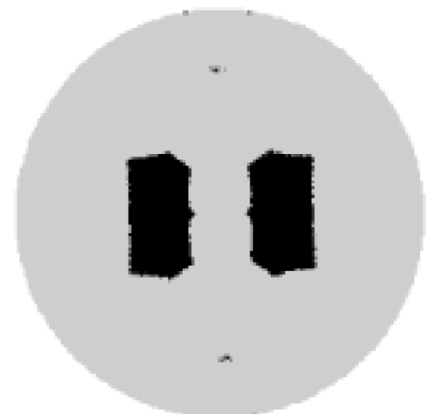

(b)

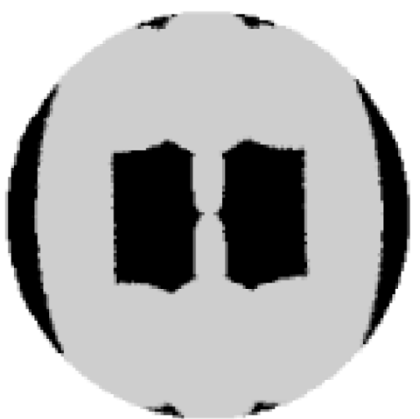

(e)

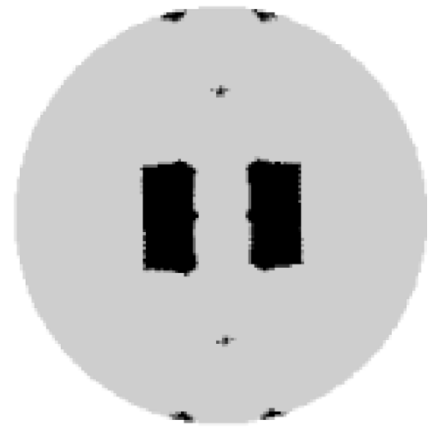

(c)

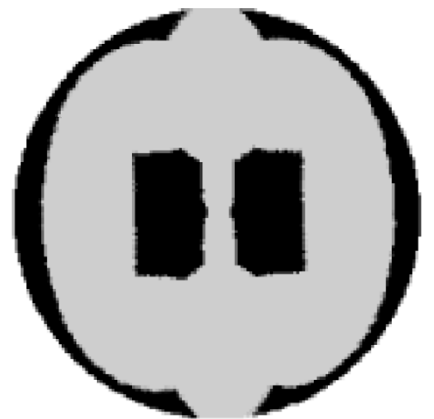

(f)

Fig. 13. Impeller position effect on the location and the shape of the rigid zones $(R e=1)$. (a) $R_{1}=0.9$ and $O d=5 ; Z R=10.6 \%$. (b) $R_{1}=0.8$ and $O d=5 ; Z R=11.69 \%$. (c) $R_{1}=0.7$ and $O d=5 ; Z R=9.1 \%$. (d) $R_{1}=0.9$ and $O d=50 ; Z R=14.57 \%$. (e) $R_{1}=0.8$ and $O d=50 ; Z R=27.48 \%$. (f) $R_{1}=0.7$ and $O d=50 ; Z R=28.85 \%$.

$$
\begin{aligned}
& -\cdot-\mathrm{Od}=50 \text { and } \mathrm{R} 1=0.9 \quad \mathrm{Od}=5 \text { and } \mathrm{R} 1=0.9 \\
& ---\mathrm{Od}=50 \text { and } \mathrm{R} 1=0.8 \quad \cdots \cdots \cdots \cdot \mathrm{O} \text { Od }=5 \text { and } \mathrm{R} 1=0.8
\end{aligned}
$$

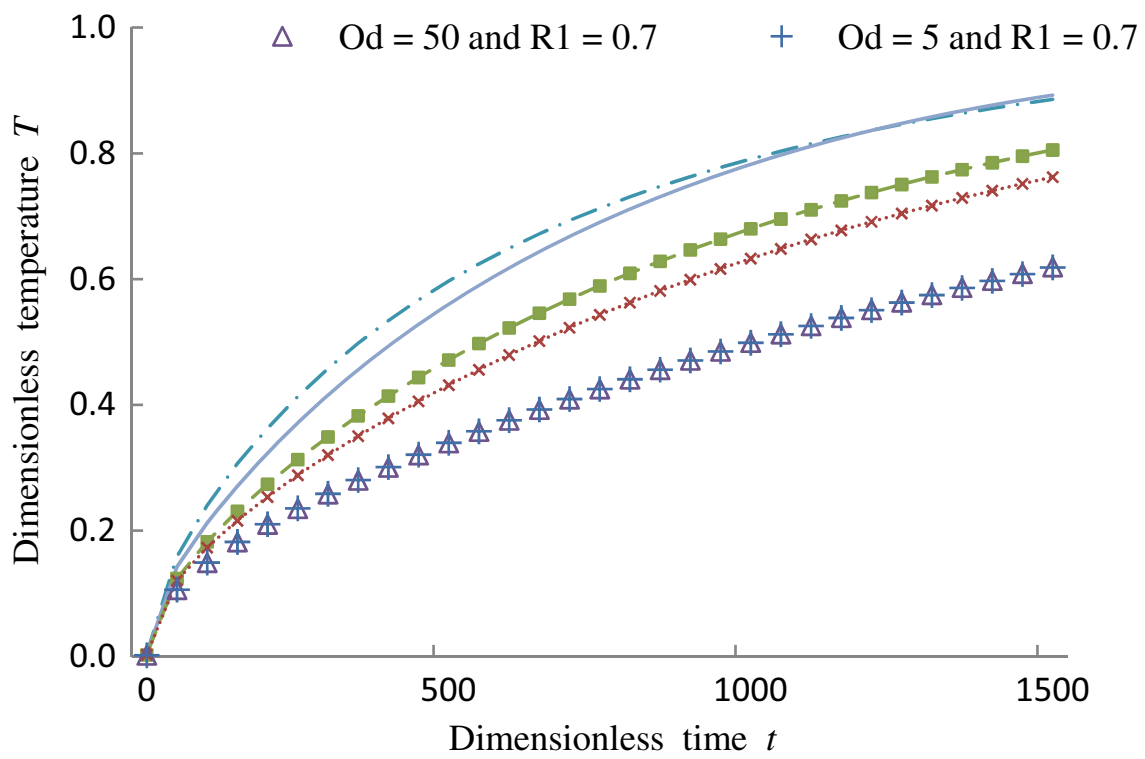

Fig. 14. Effect of the impeller position on the temporal evolution of the averaged temperature. 
were simulated to analyze the complex flow structures velocities and temperature distributions in transient regime. Indeed, resolution of coupled momentum and heat transfer equations give interesting local information concerning the hydrodynamic characteristics and the evolution with time of the thermal behaviours. The variation of the Nusselt number with the rheological parameters is also presented.

Firstly, we have put in evidence the importance of the yield stress effect on the flow structure. We have demonstrated that the area of the unyielded zones increases drastically when the Oldroyd number increases. However, the recirculation flow is intense near the impeller when the yield stress becomes important.

Secondly, it is found that the convective heat transfer is improved when the yield stress is important as a consequence of the apparition of recirculation zones. In fact, in spite of the increases of the rigid zone area, these zones are unlikely to have any repercussion for heating efficiency: the fluid is only sometimes confined in the rigid zones and then the particles can migrate outside these zones and be heated.

Thirdly, we have demonstrated that the increasing of the ratio $R_{1}$ improves heat transfer between the jacketed wall of the stirred vessel and the mixed fluid. In the particular case of $R_{1}=0.7$ and $O d=5$, we have noted that the decreasing in the rigid zone percentage masks the decreasing of the heating speed. Thus, it was noted that the reduction in rigid zone size is not obligatory the criterion of improving heating performance.

\section{References}

[1] C. Torrez, C. Andre, Simulation of a Rushton turbine mixing yield stress fluids: application of the Metzner-Otto concept, Chem. Eng. Technol. 22 (1999) 701-706

[2] P.E. Arratia, J. Kukura, J. Lacombe, F.J. Muzzio, Mixing of Shear-Thinning Fluids with Yield Stress in Stirred Tanks. AIChE J. 52 (2006) 2310-2322

[3] F. Ein-Mozaffari, S.R. Upreti, Using ultrasonic Doppler velocimetry and CFD modeling to investigate the mixing of non-Newtonian fluids possessing yield stress, Chem. Eng. Res. Des. 87 (2009) 515-523
[4] P.A. Tanguy, F. Bertrand, E. Brito, Mixing of viscoplastic fluids with anchor impellers, Chemical Engineering Symposium Series (1994) 525-532

[5] F. Bertrand, P.A. Tanguy, E. Brito, A new perspective for the mixing of yield stress fluids with anchor impellers, J. Chem. Eng. Jpn 29 (1996) 51-58

[6] D. Anne-Archard, M. Marouche, H.C. Boisson, Hydrodynamics and Metzner-Otto correlation in stirred vessels for yield stress fluids, Chem. Eng. J. 125 (2006) 15-24

[7] F. Savreux, P. Jay, A. Magnin, Viscoplastic fluid mixing in a rotating tank, Chem. Eng. Sci. 62 (2007) 2290-2301

[8] M. Baccar, M.S. Abid, Caractérisation de l'écoulement turbulent et du transfert thermique générés par des mobiles ancre et barrière dans une cuve agitée, Int. J. Therm. Sci. 38 (1999) 892-903

[9] M. Baccar, M. Mseddi, M.S. Abid, Contribution numérique à l'étude hydrodynamique et thermique des écoulements turbulents induits par une turbine radiale en cuve agitée, Int. J. Therm. Sci. 40 (2001) 753-772

[10] G. Delaplace, C. Torrez, J.C. Leuliet, N. Belaubre, C. André, Experimental and CFD simulation of heat transfer to highly viscous fluids in an agitated vessel equipped with a non standard helical ribbon impeller, Trans. IChemE 79 Part A (2001) 927-937

[11] M. Tutar, F. Erdogdu, Numerical simulation for heat transfer and velocity field characteristics of two-phase flow systems in axially rotating horizontal cans, J. Food Eng. 111 (2012) 366-385

[12] F. Erdogdu, M. Tutar, A computational study for axial rotation effects on heat transfer in rotating cans containing liquid water, semi-fluid food system and headspace, Int. J. Heat Mass Trans. 55 (2012) 3774-3788

[13] T.C. Papanastasiou, Flows of materials with yield, J. Rheology 31 (1987) 385-404

[14] S.V. Patankar, Numerical heat transfer and fluid flow, Series in Computational Methods in Mechanics and Thermal Sciences, McGraw Hill, New York, 1980

[15] J.E. Douglass, J. Gunn, A general formulation of alternating-direction implicit methods, Numer. Math. 6 (1964) 428 